\title{
LA INSTAURACIÓN DE UNA ESCUELA CO-EDUCATIVA ¿CONTRIBUYE A UNA ESCUELA MENOS DISCRIMINATORIA?, REFLEXIONES SOBRE EL CASO CHILENO.
}

\author{
Paloma Abett de la Torre Díaz \\ Condell 343-Providencia-Santiago \\ pabett@academia.cl \\ Universidad Academia de Humanismo Cristiano \\ Chile
}

\section{Resumen}

Desde los 90' Chile ha vivido un importante proceso de reformas educativas que han afectado a todo el sistema educativo. Se han hecho esfuerzos por incorporar la perspectiva de género. Sin embargo, existe el consenso que la escuela no genera los roles y estereotipos de género, pero si es el lugar donde se potencian. Frente a la premisa que la escuela es un espacio donde se discrimina, pocos se atreverían a negarlo, pero incorporar en este análisis la variable de género produce controversias ¿la escuela es un lugar que discrimina a niñas y mujeres? Sobre este complejo escenario social nos proponemos problematizar sobre qué rol podemos tener el profesorado en la instauración de una escuela co-educativa.

Palabras claves: reformas educativas, educación, género, escuela co-educativa.

\section{Abstract}

Since the 90 ' Chile has lived an important process of educational reform that has affected the whole educational system. Efforts have been made to incorporate the perspective of gender. Nevertheless, there is consensus about the fact that schools do not generate the roles and stereotypes of gender, but the place where they are promoted. If we face the premise that schools are spaces where they are discrimination occurs, few would dare to deny it. Incorporating in this analysis the variable of gender produces controversies. Is school a place that discriminates against girls and women? On this complex social scene we propose to discuss on the role we teachers must have in the restoration of a coeducational school.

Key-words: educational reforms, education, gender, coeducational school. 
Paloma Abett de la Torre Díaz. La instauración de una escuela co-educativa ¿contribuye a una escuela menos Discriminatoria?, reflexiones sobre el caso chileno.

\section{Introducción}

A principios de los años 90', Chile comienza a vivir un proceso de profunda transformación política y social, que en lo educativo, se materializa con la implementación de una Reforma Educativa. Ante el crítico diagnóstico de investigaciones publicadas como "El liceo por dentro" o "La enseñanza media en crisis", unidas a las exigencias internacionales marcadas por los tratados de libre comercio y los desafíos que implica enseñar en la sociedad del conocimiento, nuestro país comienza una reestructuración de todos los niveles de enseñanza. Dicho proceso no fue un hecho aislado, sino que muchos países de la región, como Argentina, Uruguay, Colombia, Perú, entre otros, también realizan transformaciones significativas en sus respectivos sistemas educativos.

Esta reforma se caracterizó por una nueva formulación de planes y programas, un curriculum que pretende cambiar el foco desde el contenido hacia habilidades, nuevas exigencias para el profesorado, alargamiento de la jornada escolar, entre otras. La reforma tuvo por objetivos mejorar principalmente la cobertura, equidad y calidad. Pero dichas innovaciones -necesarias sin dudas- una vez más relegaron a un segundo plano las relaciones y estereotipos de género que en la escuela se promueven.

A juicio de investigadoras como Elizabeth Guerrero, la vinculación entre género y educación no ha sido un ámbito del debate sobre educación en Chile, como tampoco ha sido una prioridad para los estudios de género o feministas, como si lo fue a fines de los ochenta y principios de los noventa. Lo que se pretende destacar es que no solo no ha sido prioridad en la agenda gubernamental, sino que ha habido una ausencia en los debates y demandas que desde la sociedad civil se podrían generar. Al parecer, existe la sensación que la igualdad en el ámbito educativo y social ha sido una meta alcanzada, que podríamos resumir con la frase tantas veces escuchada durante el último periodo presidencial "y que más quieren, si ya tienen presidenta”. 
Paloma Abett de la Torre Díaz. La instauración de una escuela co-educativa ¿contribuye a una escuela menos Discriminatoria?, reflexiones sobre el caso chileno.

Si hacemos un breve recorrido sobre las estadísticas educacionales, efectivamente ha habido avances que no se pueden desconocer, principalmente en los ámbitos de acceso y rendimiento. A modo de ejemplo, según el Análisis del Sistema Educativo Chileno desde la Perspectiva de Género, realizado por Pamela Ugalde, en base a las estadísticas e indicadores oficiales elaborados por el Ministerio de Educación, afirma que "podemos señalar que hombres y mujeres han equiparado su acceso a bienes educativos: a lo largo del tiempo han llegado a obtener similares niveles de escolaridad, no hay un patrón por género en términos del logro de aprendizajes ni una línea clara en cuanto a la matricula en enseñanza básica y media" (Ugalde 2008, p.11). De hecho en Chile, la mujer tiene en promedio más años de estudios que los hombres (11,7 frente a 10,8), por lo tanto las brechas que hay son al parecer de carácter cultural.

Esto viene a corroborar la idea que la igualdad entre hombres y mujeres ya se alcanzó. Sin embargo, pocos estudios hay referentes a las nuevas brechas que han ido surgiendo, en este contexto educativo, marcado por la sociedad del conocimiento, como es la llamada brecha digital. ¿Qué esta haciendo la escuela para revertir estos resultados?, ¿cómo explicar que en la prueba SIMCE$^{1}$ las mujeres siguen obteniendo mejores resultados en lenguaje y los hombres en matemáticas?, ¿cómo entender que nuestras estudiantes obtienen mejores notas durante sus años de escolarización ${ }^{2}$, repiten en menor cantidad que los varones y al verse enfrentadas a la prueba de selección universitaria, obtienen puntajes más bajos que sus compañeros? Los anteriores cuestionamientos nos ayudan a comprender que es necesario realzar el tema de las relaciones de género en el análisis de la desigualdad en la educación escolar.

Hacerse cargo de estas problemáticas, implica incorporar la variable de géneroentendiendo por tal, la construcción cultural de la diferencia sexual- en el análisis del curriculum oculto que se transmite en nuestro sistema educativo, pero también en el curriculum explicito que valora y reconoce a unos y otras de manera desigual. 
Paloma Abett de la Torre Díaz. La instauración de una escuela co-educativa ¿contribuye a una escuela menos Discriminatoria?, reflexiones sobre el caso chileno.

No se puede desconocer el esfuerzo realizado durante la segunda mitad de los 90', por hacer visibles a las mujeres que emergieron tanto del Servicio Nacional de la Mujer, como del Ministerio de Educación ${ }^{3}$, cuyo principal logro ha sido la utilización de lenguaje inclusivo y la revisión de textos escolares, en los cuáles se ha promovido la incorporación del enfoque de género. Especial importancia han tenido el Plan de Igualdad de Oportunidades para las Mujeres 1994-1999, el Plan de Igualdad de Oportunidades entre Mujeres y Varones 2000-2010, como también, la Estrategia de transversalización de género en la administración pública compuesta por los Compromisos Ministeriales y Sistema de Género del Programa de Mejoramiento de la Gestión (PMG de género). Estas iniciativas han permitido introducir y visibilizar el tema en el MINEDUC (como también en otras reparticiones del Estado), pero no se ha logrado elaborar una política de género a nivel nacional ni tampoco en el Ministerio de Educación. Consideramos que para tener un sistema educativo más equitativo en cuanto a relaciones de género, es necesario, reformular, nuestra escuela mixta.

\section{La escuela mixta: ¿es igual para todas y todos?}

La escuela mixta que emerge a principios del siglo XX en nuestro país, debido a la urgencia y principalmente a la escasez de recursos, ya que en muchos lugares se hacía dificultoso financiar escuelas para hombres y mujeres. Ha ido desarrollándose como el y único modelo educativo. Hace ya tiempo que no tenemos curriculums diferenciado para niñas y niñas, por lo menos en las escuelas públicas, es por ello, que cuesta criticar a la escuela mixta, que parte de la premisa que al estar todos y todas juntos, se enseña y aprende lo mismo.

"El modelo de escuela mixta es el que tenemos en la actualidad, en la que existe igualdad formal en todos los aspectos pero no igualdad real. La escuela mixta cree tratar y atender a todo el alumnado por igual. Intenta fomentar el desarrollo de todas las capacidades personales. No contempla la importancia de los géneros. Respeta la libertad de elección. Prepara a los individuos para el mundo laboral. Cree en el principio de que el alumnado obtiene el éxito de acuerdo con sus 
Paloma Abett de la Torre Díaz. La instauración de una escuela co-educativa ¿contribuye a una escuela menos Discriminatoria?, reflexiones sobre el caso chileno.

méritos. Contempla un modelo de alumno ideal. Parte del principio de la neutralidad escolar" (Tomé 1999, p. 174).

Sin embargo, frente a los resultados y logros educativos, anteriormente señalados o a la elección profesional de nuestros estudiantes emergen nuevas preguntas como “¿hasta qué punto es suficiente con agrupar en un solo escenario escolar a las niñas y a los niños? ¿Es la escuela un lugar donde se fomenta la igualdad de derechos y de oportunidades o, por el contrario, un escenario en el que el orden simbólico sigue siendo masculino y se ocultan los deseos, los saberes y las formas de vida de las niñas y de las mujeres? ¿Cómo son las interacciones en el aula? ¿Quién habla a quién, cómo, cuánto, cuándo y de qué manera? ¿Cómo se seleccionan los contenidos escolares en los currículos y en los libros de textos y en qué medida tienen en cuenta las expectativas de las niñas y la autoridad simbólica de las mujeres? ¿Contribuye el sistema educativo a la difusión de los estereotipos de género? ¿Oculta el lenguaje de la escuela a las niñas y a las mujeres? ¿Es posible cambiar la educación sin que ello afecte a las asimetrías de género?’ (Lomas 1999, p. 10).

Los estudios sobre este tema aseguran que los mayores grados de discriminación de género se dan en las escuelas mixtas, ya que el profesorado tiende a perpetuar en el sistema educativo los roles que la sociedad ha impuesto a hombres y mujeres. Es en la escuela mixta donde se asume que las niñas son buenas en lenguaje y arte, pero no para las ciencias. El curriculum en asignaturas como educación física siegue siendo segregado y en matemática los varones continúan obteniendo mejores resultados.

Otros fenómenos que se producen en la escuela mixta, que se deben estudiar y analizar es que "A los niños aún se los educa para poder ejercer la violencia, como si tuvieran que enfrentarse diariamente a terribles peligros físicos. $Y$ a las niñas se las educa en la atención a la belleza, como si su futuro siguiera dependiendo de sus posibilidades en el mercado matrimonial' (Subirats 1999, p. 19). A modo de ejemplo, el estudio Informe Nacional sobre Violencia de Género 
Paloma Abett de la Torre Díaz. La instauración de una escuela co-educativa ¿contribuye a una escuela menos Discriminatoria?, reflexiones sobre el caso chileno.

en la Educación Básica en México, destacó que El 50.1\% de los niños de primaria está de acuerdo con que "el hombre es el que manda y decide lo que le conviene a la familia" frente al $31.7 \%$ de las niñas que opina lo mismo. El $79.2 \%$ de las niñas y los niños está de acuerdo en que "el hombre es el que debe tener la mayor responsabilidad para traer el dinero al hogar", y en que las niñas deben aprender a cuidar a sus hermanitos y haciendo limpieza. Asimismo el estudio demostró que el $60.3 \%$ de los adolescentes de secundaria y el $54.8 \%$ de las adolescentes en este nivel, está de acuerdo en que "la mujer es la que tiene que cuidarse para no quedar embarazada"”.

Para Marina Subirats, la escuela mixta tan masificada y alabada no ha conseguido, la igualdad en la educación de hombres y mujeres, puesto que si bien niños y niñas se educan en los mismos establecimientos, el modelo pedagógico dominante sigue teniendo un carácter androcéntrico, entendiendo por tal una visión de mundo que pone al hombre como centro y medida de todas las cosas. "Esta visión del mundo y de las cosas parte de la idea de que la mirada masculina es una mirada universal y objetiva que se ejerce en nombre de la humanidad. El androcentrismo conlleva la invisibilidad de las mujeres y la ocultación de sus aportaciones tanto en los ámbitos de la vida cotidiana de las sociedades como en los contextos de las ciencias, el saber y de las artes. El androcentrismo constituye una visión distorsionadora y empobrecedora de la realidad que oculta las relaciones de poder y de opresión edificadas a lo largo de los siglos por el poder simbólico masculino contra las mujeres. El androcentrismo supone también la imposición de maneras únicas y arquetípicas de <<ser>> hombres y mujeres" (Lomas 2004, p. 230).

A modo de ejemplo, como diversos estudios han demostrado "sabemos hoy que el profesorado no se comporta del mismo modo ante las chicas que ante los chicos, pues tiende a creer que las niñas son más constantes y menos intuitivas que los niños, más ordenadas, más trabajadoras, más responsables, más maduras, con menor capacidad creativa, más pasivas, menos audaces, menos dotadas para las supuestas disciplinas científicas y técnicas, y más interesadas por la literatura o la enseñanza doméstica” (Rodríguez 2007, p. 21). 
Paloma Abett de la Torre Díaz. La instauración de una escuela co-educativa ¿contribuye a una escuela menos Discriminatoria?, reflexiones sobre el caso chileno.

Si remiramos cuanto de la visión androcéntrica sigue perdurando en la escuela mixta, podremos entender las grandes diferencias que hay en el conocimiento de las ciencias, o por qué a pesar de los avances de la historia de las mujeres o de la historia social, perviven los héroes, como agentes del cambio social.

Asimismo queremos destacar que para la implantación de una escuela co-educativa, es necesario que se comprenda que este proyecto no es una acción a desarrollar por mujeres, que están disconformes con la escuela mixta, sino que frente al cúmulo de información con que hoy se cuenta, creemos que este tipo de escuela, reproduce estereotipos de género limitantes y restrictivos para ambos sexos, es decir, a los hombres el modelo de escuela mixta también los afecta. Para revertir y no seguir perpetuando esta situación proponemos el desarrollo de una escuela co-educativa.

\section{Repensado una nueva escuela: ideas para la instauración de la escuela co-educativa}

Frente a las desigualdades que la escuela mixta ha generado, emerge un tipo de modelo que se denomina escuela co-educativa, el que se caracteriza porque "presupone que el profesorado reconoce las forma con las que el sexismo se manifiesta en el centro e incluso llega a reconocerse como parte implicada en la producción de esa desigualdad. La escuela es una institución que contempla la eliminación de las desigualdades sexuales y de las jerarquías de género. Atiende las necesidades de los grupos más que las de los individuos y a las diversidades culturales. Reconoce la no neutralidad escolar. Crea nuevos ordenes discursivos, temporales y espaciales. Prepara a los individuos para el mundo laboral y la esfera privada y familiar" (Tomé 1999, p. 174).

Si pretendemos generar una educación igualitaria y equitativa para hombres y mujeres, un factor clave es repensar la formación inicial docente. Pues actualmente en Chile, no existe una formación que incorpore de manera transversal la perspectiva de género, esta se remite 
Paloma Abett de la Torre Díaz. La instauración de una escuela co-educativa ¿contribuye a una escuela menos Discriminatoria?, reflexiones sobre el caso chileno.

principalmente a ramos optativos ${ }^{5}$. Sabemos que las demandas con respecto a qué tipo de formación debe tener la docencia del siglo XXI es variopinta, pasando desde educación en nuevas tecnologías, interculturalidad e idiomas, pero consideramos que incluir en ello el enfoque de género, contribuiría ha mejorar la calidad de nuestra educación. Entendiendo que este enfoque, no es propio de una disciplina, sino que debe ser trabajado, desde el curriculum y las prácticas docentes. En Chile los docentes hombres duplican a las mujeres en educación media científicohumanista, en la educación media técnico-profesional esta brecha se acrecienta, esta constatación ¿nos ayudaría a comprender, porque se sigue impartiendo mecánica y electricidad para hombres; y secretariado y técnico en alimento para mujeres? ${ }^{6}$.

El profesorado en Chile es mayoritariamente femenino, "en términos concretos, del total de 176.472 docentes el 71 por ciento son mujeres respecto al $29 \%$ de hombres. Es decir 2 de cada 3 docentes del sistema escolar son mujeres" (Mineduc, 2008). Al analizar otros niveles del sistema educativo, el panorama se invierte, si se compara la participación de hombres y mujeres en el sistema parvulario, las mujeres representan el 98\%. "Así entonces, vemos que las mujeres se sitúan en nichos dedicados al cuidado infantil y reemplazo de la madre al interior de las escuelas. Los hombres, en cambio, se dedican a la transmisión de conocimientos (atienden alumnos de más edad) y a la enseñanza de un oficio, reproduciendo el rol de padre proveedor: así como el padre hereda a su hijo su dinero y posesiones, la tradición de su familia y su oficio, el maestro varón lo hace en la escuela, es él quien habilita al niño para incorporarse prontamente al mercado laboral. Las maestras, en cambio habilitan a las niñas en conocimiento científico, letras y humanidades, herramientas que no las acercan al mundo del trabajo sino que las mantienen en la esfera de lo doméstico" (Ugalde 2008, p. 12).

Esta diferenciación sobre los saberes e ideas transmitidas en la escuela, que después repercuten en la elección de estudios superiores o en las ocupaciones que ha futuro desempeñamos las personas, sólo pueden desentrañarse desde un análisis de género. Como destaca la especialista en género y educación, Sandra Acker los "códigos de género o currículo 
Paloma Abett de la Torre Díaz. La instauración de una escuela co-educativa ¿contribuye a una escuela menos Discriminatoria?, reflexiones sobre el caso chileno.

oculto no concluyen con tanta facilidad culpando al profesorado como lo hacen los estudios de interacción en el aula, ya que los procesos de la escuela parecen lo suficientemente hegemónicos como para obligarles a corregir sus errores. La acción del profesorado parece enraizada en la tradición y en la rutina más que en la malicia. Sin embargo, surge una pregunta legítima sobre cómo, cuándo y por qué las profesoras retan a esta hegemonía o, contrariamente, por qué a menudo no lo hacen. Estas preguntas nos llevan a centrarnos en la propia profesora, en vez de en el alumnado o en la naturaleza de la interacción entre ambos” (Acker 1995, p. 123). Es por ello, que consideramos imprescindible repensar el proceso de formación docente.

Consideramos que algunas líneas de acción que se podrían implementar en las instituciones formadoras de docentes y en las propias escuelas serían:

- Reflexionar desde una perspectiva de género y en el ámbito de la educación en especial sobre la situación que actualmente viven hombres y mujeres, en los espacios públicos como en los privados.

- Sensibilizar sobre la necesidad de la participación de las mujeres y hombres en los diferentes ámbitos sociales, políticos y laborales.

- Analizar los mecanismos discriminatorios, tanto explícitos como ocultos, que subyacen en las prácticas, contenidos y materiales educativos.

- Promover acciones de intervención práctica para una educación igualitaria y equitativa.

\section{Conclusiones}

Cuando pensamos en desarrollar estrategias para terminar con el sesgo de la escuela mixta que tanto a hombres como a mujeres afecta, necesariamente debemos cuestionarnos sobre el tipo de modelo de escuela que pretendemos construir y con que medios contamos para lograrlo. Como señala Amparo Tomé "Para que el profesorado empiece a plantearse un nuevo modelo de escuela es necesario que se pregunte: ¿qué se entiende por igualdad entre los sexos?, ¿qué se entiende por diferencia? ¿qué se entiende por jerarquía de géneros?, ¿en qué consiste la escuela mixta?, ¿qué valores se imparten en la escuela mixta?, ¿qué es la escuela coeducativa?. ¿qué 
Paloma Abett de la Torre Díaz. La instauración de una escuela co-educativa ¿contribuye a una escuela menos Discriminatoria?, reflexiones sobre el caso chileno.

tipos de estrategias se pueden utilizar para evitar el sexismo en la escuela?, ¿se pretende conseguir la igualdad entre las niñas y los niños en todos los espacios escolares?, ¿son válidas las mismas estrategias educativas hoy día para las niñas y para niños?, ¿con qué medios humanos y materiales contamos para iniciar un proceso de cambio?' (Tomé 1999, p. 173).

El modelo de escuela co-educativa, implica en primer lugar un cambio en la formación y prácticas docentes, y después en las prácticas del estudiantado. Pero aun asumiendo que el profesorado tenga presente su papel en la constitución de los géneros, necesita de una metodología para modificar su actuar, que es necesario reflexionar y planificar.

Este mundo complejo, veloz e interconectado, necesita un nuevo tipo de escuela, ha habido avances en otros ámbitos sociales, ¿por qué en términos de relaciones de género, sigue siendo tradicional? No podemos seguir teniendo una actitud pasiva frente a las desigualdades que la escuela mixta genera. Quizás el primer paso es que estemos concientes que ello ocurre, porque la escuela, al no intervenir, refuerza, fortalece, mantiene, produce y reproduce estereotipos de género. Por consiguiente la escuela puede y debe intervenir ya que contribuye a definir y redefinir los géneros.

Si pretendemos tener sociedades más integradas en términos de género, urge que las políticas públicas con respecto a la educación continúen con las exigencias de utilizar lenguaje inclusivo, evitar los sesgos de género, etc. Como asimismo que desde el campo académico, se continúen realizando investigaciones en el área. Pero sin lugar a dudas, creemos, que junto con las revisión sobre como se están formando los futuros docentes, es necesario que en las propias escuelas se reflexione sobre la temática de género. Para ello, se hace indispensable una fase de sensibilización con respecto al tema, ya que múltiples investigaciones han destacado que existe desigualdad de género en todos los centros educativos, por lo que se hace difícil imaginar que el profesorado vea la necesidad de cambiar algo que no evidencia como problema. 
Paloma Abett de la Torre Díaz. La instauración de una escuela co-educativa ¿contribuye a una escuela menos Discriminatoria?, reflexiones sobre el caso chileno.

En una segunda fase promovemos el desarrollo de un proceso de investigación-acción, que le permita a la propia comunidad educativa realizar un diagnóstico de cuáles son las situaciones críticas que viven tanto hombres y mujeres al interior del establecimiento y de este modo ser capaces de generar un respuesta. Sin embargo, sabemos que el hecho el conocer el problema no implica, por si mismo tener las soluciones para resolverlo. El cambio de prácticas sexistas es complejo porque las razones que lo explican son multicausales $y$, en definitiva el diseño de las estrategias de cambio debe estar bien elaborado, reflexionado, argumentado y secuenciado, atendiendo a las múltiples dimensiones que la problemática plantea y con la conjunción de los diferentes actores del sistema podremos superar el sesgo histórico de la escuela mixta, y comenzar a desarrollar una escuela co-educativa. 


\section{Bibliografía}

ACKER, Sandra. Género y Educación: reflexiones biológicas sobre mujeres, enseñanza y feminismo. Madrid, Ediciones Narcea, 1994.

GUERRERO CAVIEDES, Elizabeth, PROVOSTE FERNÁNDEZ, Patricia y VALDÉS BARRIENTOS, Alejandra. "Acceso a la educación y socialización de género en un contexto de reformas educativas". En: Hexagrama Consultoras, Equidad de Género y reformas educativas: Argentina, Chile, Colombia y Perú, Instituto de Estudios Sociales Contemporáneos -IESCOUniversidad Central de Colombia y Facultad de Ciencias Sociales -FLACSO- Buenos Aires, Santiago, 2006.

LOMAS, Carlos (coord.). ¿Iguales o diferentes?: Género, diferencia sexual, lenguaje y educación. Barcelona, Editorial Paidós, 1999.

LOMAS, Carlos (comp.). Los chicos también lloran: identidades masculinas, igualdad ente lo sexos y coeducación. Barcelona, Editorial Paidós, 2004.

MINEDUC. Análisis del Sistema Escolar desde la Perspectiva de Género 2008.

RODRIGUEZ, Vega. "La educación como ámbito prioritario de aplicación de la transversalidad de género". En: VEGA Navarro Ana (coordinadora). Mujer y educación: una perspectiva de género. Ediciones Aljibe, Málaga, 2007. 
Paloma Abett de la Torre Díaz. La instauración de una escuela co-educativa ¿contribuye a una escuela menos Discriminatoria?, reflexiones sobre el caso chileno.

SUBIRATS MARTORI, Marina. "Género y escuela" En: LOMAS, Carlos (coord.). ¿lguales o diferentes?: género, diferencia sexual, lenguaje y educación. Barcelona, Editorial Paidós, 1999.

TOMÉ GONZÁLEZ Amparo. "Un camino hacia la coeducación (Instrumentos de reflexión e intervención) En: LOMAS, Carlos (coord.). ¿lguales o diferentes?: género, diferencia sexual, lenguaje y educación. Editorial Paidós, Barcelona, 1999.

UGALDE, Pamela. Análisis Del Sistema Educativo Chileno Desde La Perspectiva de Género año 2005, Ministerio de Educación, División de Planificación y Presupuesto Departamento de Estudios y Desarrollo, Enero 2008.

VV.AA. Género y educación: la escuela coeducativa. Barcelona, Editorial Graó, 2002. 
${ }^{1}$ Como destaca la Organización de Estados Iberoamericanos. [en línea]. En 4ํㅡásico, los puntajes promedio muestran diferencias por género en la prueba de Lectura a favor de las mujeres, quienes además aumentan significativamente su puntaje promedio nacional en relación al año 2007. No se presentan diferencias por género en la prueba de Educación Matemática de $4^{\circ}$ Básico. En $2^{\circ}$ Medio, se observan diferencias significativas por género para los dos subsectores evaluados. Estas diferencias favorecen a las mujeres en Lengua Castellana y Comunicación, y a los hombres en Matemática. http://www.oei.es/noticias/spip.php?article4912 [consulta: 1 de Julio 2010].

${ }^{2}$ Ver estudio MINEDUC, [en línea]. Análisis del Sistema Escolar desde la Perspectiva de Género 2008.

En http://ded.mineduc.cl/mineduc/ded/documentos/Analisis de Sistema Escolar desde la Perspecti va de Genero 2008.pdf [consulta: 2 de Julio 2010 ].

${ }^{3}$ Se puede destacar, a nivel preescolar la incorporación de la dimensión de género en las Bases Curriculares de la Educación Parvularia. En la educación básica la Incorporación de Principios de Igualdad de Oportunidades, Decreto 240, con propuestas con contenidos de género en los programas de $5^{\circ}, 6^{\circ}, 7^{\circ}$ y $8^{\circ}$ Básico. En la Enseñanza Media la Incorporación de Principios de Igualdad de Oportunidades, Decreto 220 , con contenidos de género en los programas de $1 \stackrel{\circ}{\circ} 2^{\circ}, 3^{\circ} \mathrm{y}$ $4^{\circ}$ año de educación media.

${ }^{4}$ Para mayor información ver México: La educación tiene un papel central en la prevención de la violencia de género: Lujambio [en línea] en http://www.unicef.org/mexico/spanish/mx $\mathrm{pr}$ SEPUNICEF VIOLENCIA DE GENERO.pdf

${ }^{5}$ Ver estadísticas SERNAM Existencia de cátedras sobre perspectiva de género.

${ }^{6}$ Según el documento Análisis del Sistema Escolar desde la Perspectiva de Género 2008 [en línea]. En la Enseñanza Media Técnica Profesional las mujeres presentan mayor porcentaje de participación en los sectores económicos de Administración y Comercio, Alimentación, Hotelería y Turismo. Su presencia disminuye es en el sector Metalmecánico, Minero, Química, donde la 
presencia de profesoras es casi nulo. La docencia masculina se concentra en Administración y Comercio, en Metalmecánico y en Electricidad.

http://w3app.mineduc.cl/DedPublico/estudios recientes 